
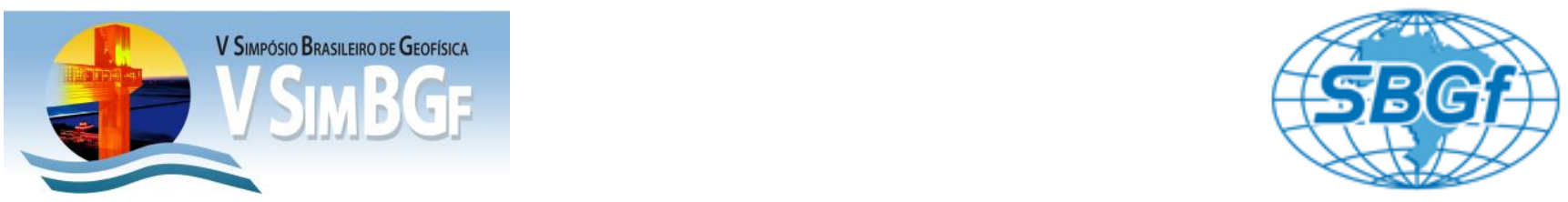

\title{
Sondagem elétrica vertical aplicada na estimativa de dimensões verticais de unidades geológicas no norte da cidade de Sinop - MT
}

Rafaela leite Jansons (ICET/UFMT); Djonatan Freitas dos Santos* (ICET/UFMT), Alterêdo Oliveira Cutrim (ICET/UFMT), Ricardo Cortês Guimarães (ICET/UFMT), Cassiana Lussi(ICET/UFMT).

Copyright 2012, SBGf - Sociedade Brasileira de Geofísica

Este texto foi preparado para a apresentação no V Simpósio Brasileiro de Geofísica, Salvador, 27 a 29 de novembro de 2012. Seu conteúdo foi revisado pelo Comite Técnico do V SimBGf, mas não necessariamente representa a opinião da SBGf ou de seus associados. É proibida a reprodução total ou parcial deste material para propósitos comerciais sem prévia autorização da SBGt.

\section{Abstract}

Through the technique of VES (vertical electrical sounding) were five VES with maximum aperture of $A B / 2$ $1,000 \mathrm{~m}$ in the northern city of Sinop - MT, to determine the thickness of the soil and the depth and thickness of geological units. The results of VES allowed to correlate stratigraphically from bottom to top the Dardanelos Formation, Salto das Nuvens , Utiariti and Pedological cover. The Dardanelos Formation has a thickness greater $100 \mathrm{~m}$ and depth of between $325 \mathrm{~m}$ and $400 \mathrm{~m}$, the Salto das Nuvens Formation has a thickness ranging from $260 \mathrm{~m}$ to $350 \mathrm{~m}$ and depth of $49,9 \mathrm{~m}$ and $89,95 \mathrm{~m}$, and
Utiariti Formation has a thickness of $45 \mathrm{~m}$ and $86 \mathrm{~m}$, depth between $1,6 \mathrm{~m}$ and $6,4 \mathrm{~m}$ and the soil has thickness ranging from $1,6 \mathrm{~m}$ to $6,4 \mathrm{~m}$.

\section{Introdução}

O abastecimento de água no município de Sinop é feito pela exploração do manancial subterrâneo, no entanto o conhecimento sobre essas águas é limitado pelo pouco conhecimento sobre as dimensões verticais do solo e das formações geológicas. Desse modo aplicou-se a técnica de Sondagem Elétrica Vertical (SEV) para estimar a profundidade e espessuras das unidades geológicas que constituem a estratigrafia da área, dada a eficiência dessa técnica para tal finalidade (Cutrim e Rebouças, 2005; Cutrim, et al., 2007; Cutrim e Shiraiwa, 2011). A área de estudo tem $78 \mathrm{~km}^{2}$ e está situada no centro-norte do estado de Mato Grosso e compreende a porção norte da cidade de Sinop, a 500km da capital Cuiabá (figura 1).
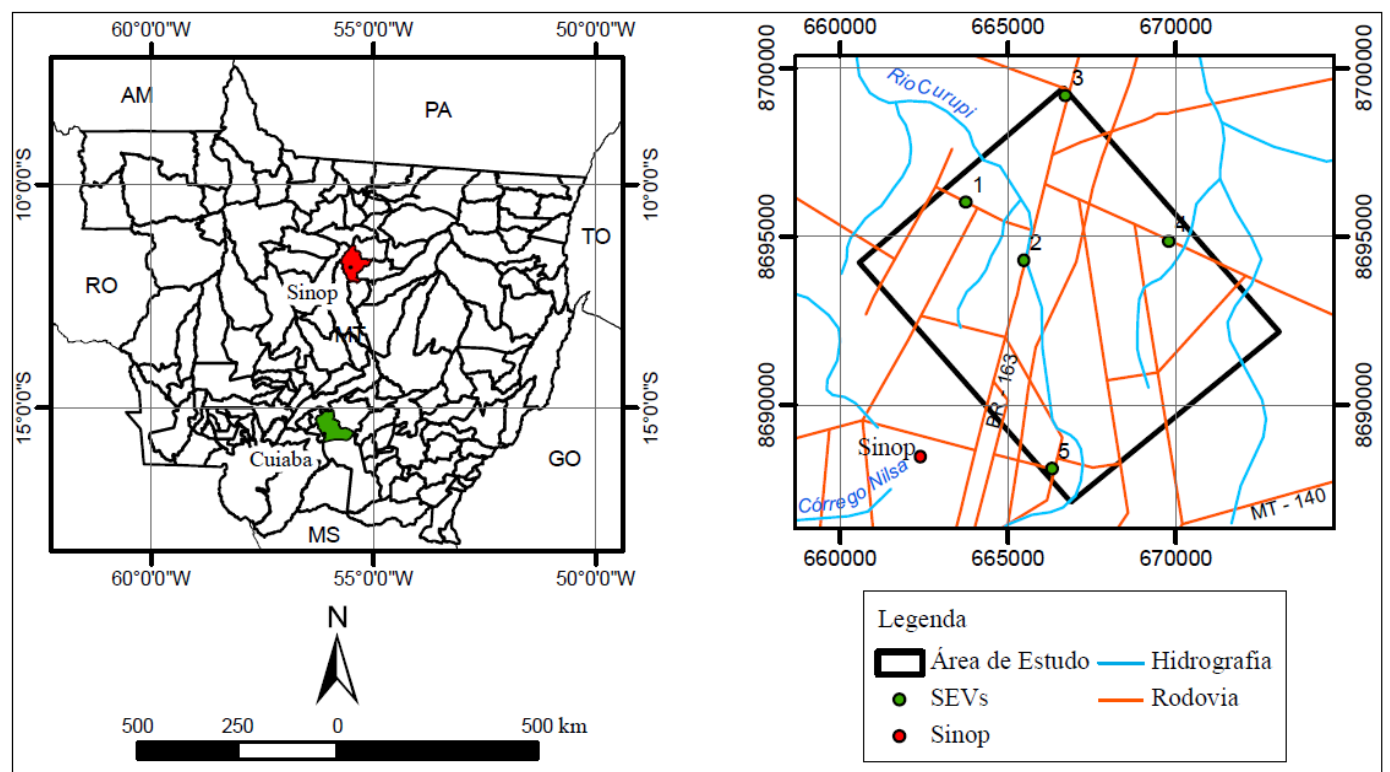

Figura 1 - Mapa de Localização com o posicionamento das SEVs na área de estudo.

\section{Geologia e Hidrogeologia da Área}

Segundo Padilha et al. (1974) a área de estudo insere-se na Bacia do Parecis, constituída pelo Grupo Parecis (Formações Salto das Nuvens e Utiariti) e pela Formação Dardanelos. A Formação Dardanelos é composta por arenito com raros níveis de conglomerado. A Formação Salto das Nuvens é constituída por conglomerado, arenitos grosso e fino, pelito e argilito e argilito calcífero. A Formação Utiariti é composta por arenitos finos a médios bem selecionados. A área é coberta essencialmente por Latossolos vermelhos e amarelos 
(PRODEAGRO, 2001). Os principais aquíferos da área são o Utiariti, Salto das Nuvens e Dardanelos, sendo os dois primeiros os únicos explorados (RIBEIRO, 2009).

\section{Metodologia}

No presente trabalho foi utilizada a técnica de SEV (Sondagem Elétrica Vertical) que permite investigar a variação da resistividade no plano vertical. A técnica consiste na injeção de corrente elétrica (I) no meio através de dois eletrodos ( $\mathrm{A}$ e $\mathrm{B}$ ) e medida da diferença de potencial $(\Delta V)$ entre dois eletrodos $(\mathrm{M}$ e $N)$ localizados entre os eletrodos de corrente. Como a corrente flui de modo radial, quanto maior a distância entre $(A)$ e $(B)$, maior será a profundidade investigada. A resistividade é obtida através de $(\mathrm{I})$ e $(\Delta \mathrm{V})$, conforme abaixo Bhattacharya e Patra (1968):

$$
\rho_{a}=\left(\frac{\Delta V}{I}\right) \cdot \frac{2 \pi}{\frac{1}{\mathrm{AM}}-\frac{1}{\mathrm{BM}}-\frac{1}{\mathrm{AN}}+\frac{1}{\mathrm{BN}}}
$$

Foram realizadas cinco SEVs (figura 1), com $A B / 2$ máximo de $1.000 \mathrm{~m}$ e $\mathrm{MN} \leq \mathrm{AB} / 5$, usando o arranjo Schlumberger e um resistivímetro com $250 \mathrm{~W}$ de potência e voltagem máxima de $800 \mathrm{~V}$. As SEVs foram ajustadas usando o método Ridge Regression (Tikhonov e Arsenin, 1977).

\section{Resultados e Discussões}

As Figuras 2, 3. 4, 5 e 6 mostram o padrão de curva de resistividade adotado para as SEVs modeladas pelo Software IPI2win e os resultados da modelagem e interpretação das cinco SEVs estão apresentados na Tabela 1.

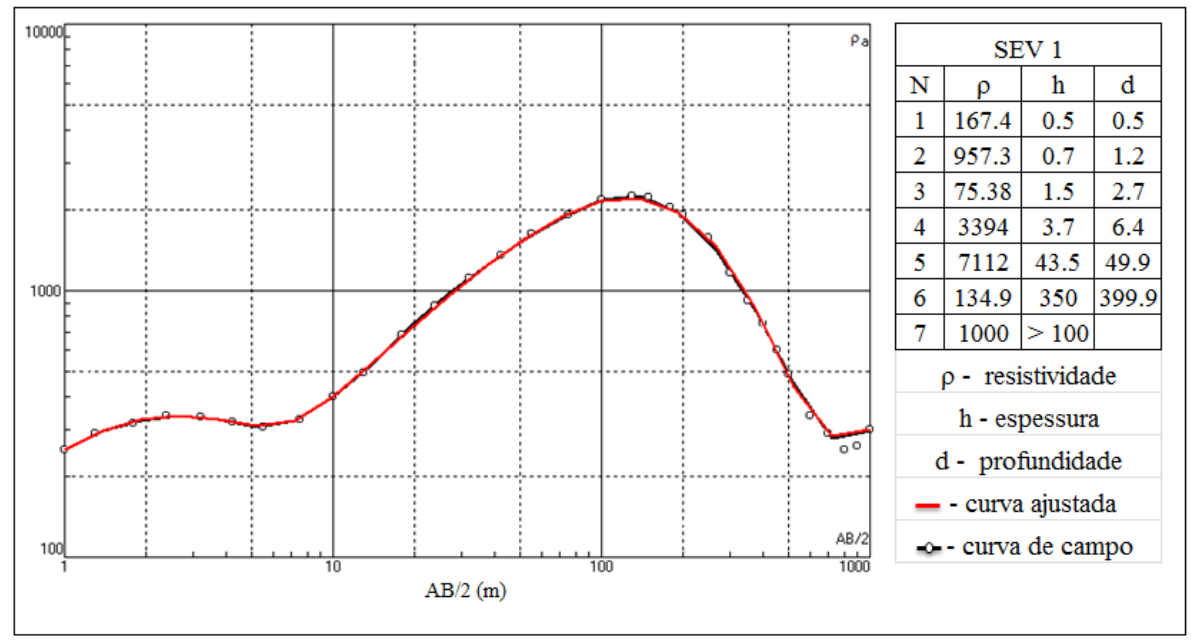

Figura 2 - Perfil geoelétrico da SEV 1.

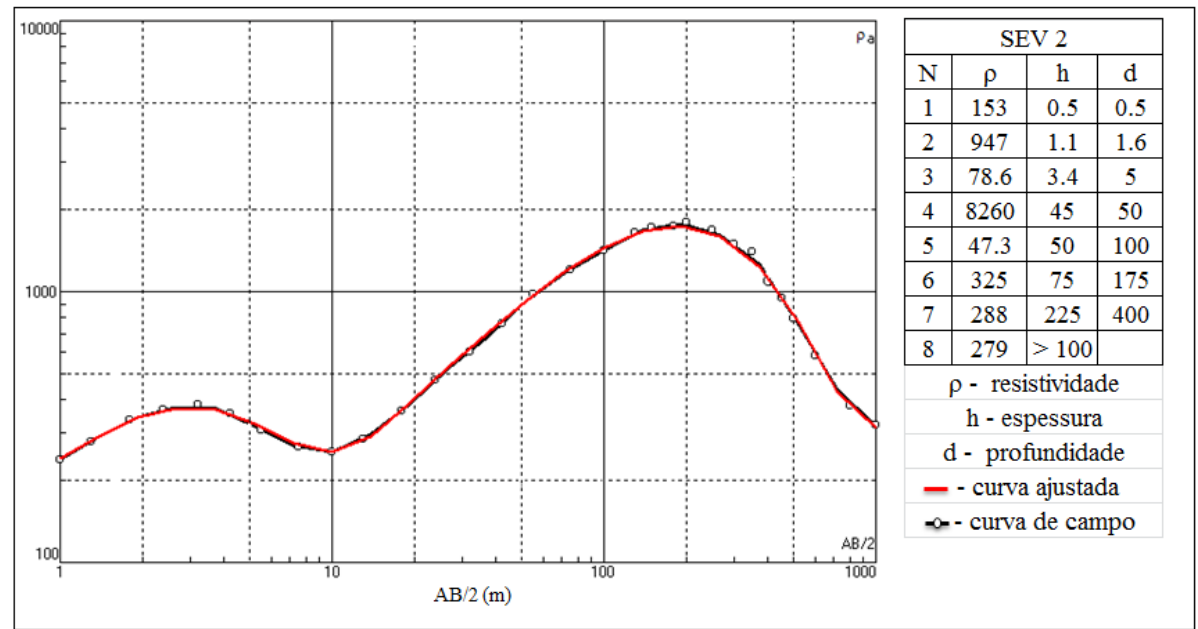

Figura 3 - Perfil geoelétrico da SEV 2. 


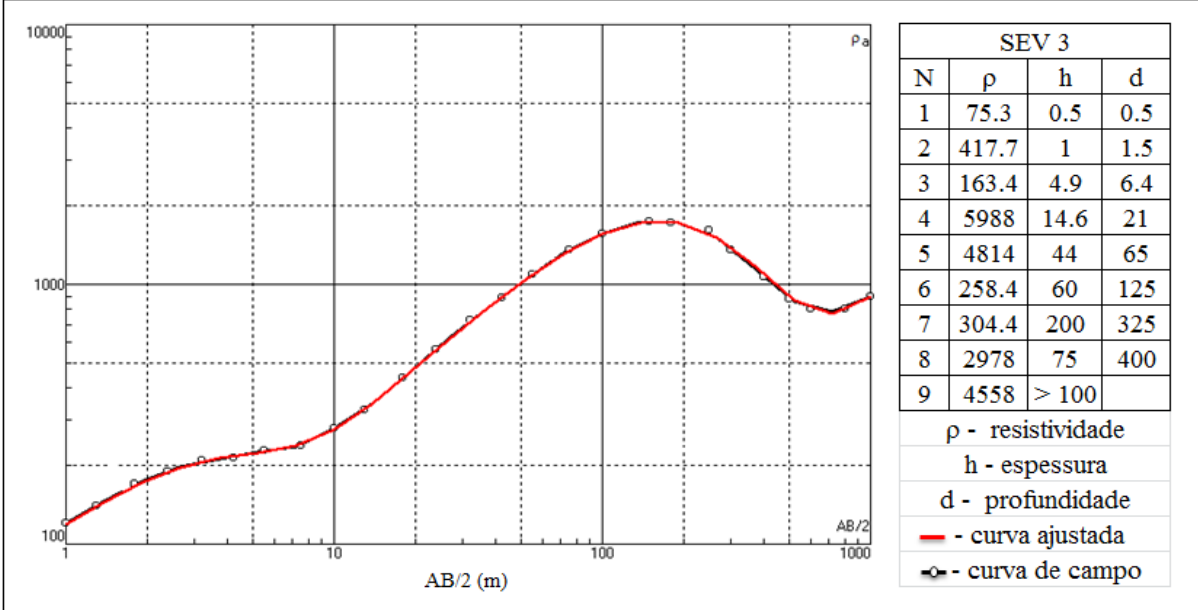

Figura 4 - Perfil geoelétrico da SEV 3.

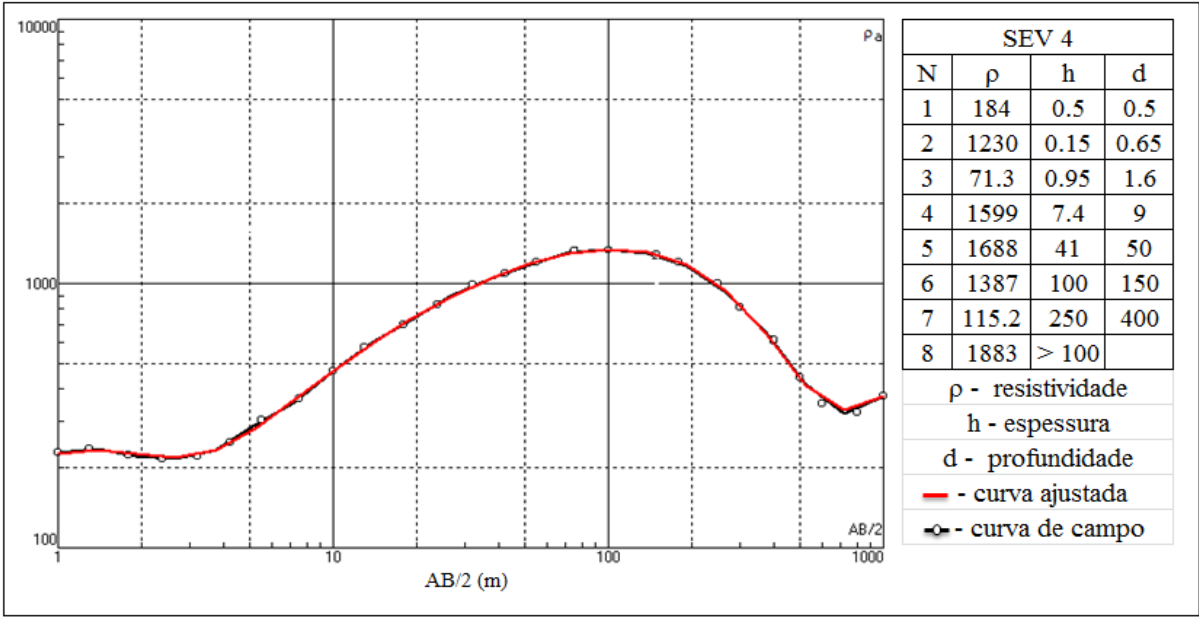

Figura 5 - Perfil geoelétrico da SEV 4.

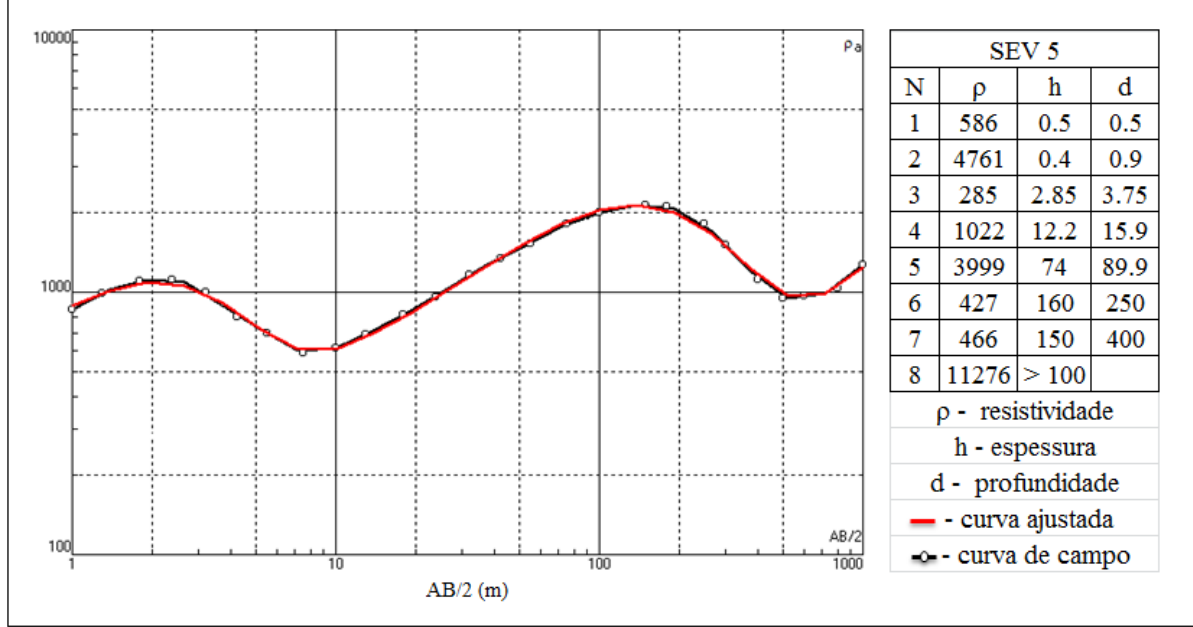

Figura 6 - Perfil geoelétrico da SEV 5. 
Em todas as SEVs, as três primeiras camadas representam a cobertura pedológica, com resistividades variando entre $71,3 \Omega \mathrm{m}$ e $4.761 \Omega \mathrm{m}$ e espessuras entre $1,6 \mathrm{~m}$ a $6,4 \mathrm{~m}$. A variação da resistividade sugere heterogeneidade do solo e atividade antrópica. As camadas 4 e 5, com exceção da SEV 2 (apenas camada 4), apresentam resistividades mais altas, entre $1.022 \Omega \mathrm{m}$ a $8.260 \Omega \mathrm{m}$, com espessura variando de $45 \mathrm{~m}$ a $86,2 \mathrm{~m}$ e correspondem à Formação Utiariti. $O$ aumento das resistividades se deve à composição arenítica dessa unidade geológica.
A Formação Salto das Nuvens é representada pelas camadas 6 e 7 (exceção as SEV 1 e 2, que são representadas pelas camadas 6 e 5,6 e 7 , respectivamente), com profundidade ao topo de 49,9 $\mathrm{m}$ a

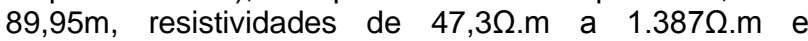
espessura de $260 \mathrm{~m}$ a $350 \mathrm{~m}$. A camada 8 (exceção às SEV 1, representada pela camada 7 , e a SEV 3, representada pelas camadas 8 e 9), correspondem à Formação Dardanelos com resistividades maiores que $1.000 \Omega$.m, e espessuras maiores que $100 \mathrm{~m}$, com exceção da SEV 3 que apresenta espessura maior que $175 \mathrm{~m}$.

Tabela 1 - Espessura, profundidade, resistividade e coordenadas (Sistema WGS84) produzidas pela modelagem das SEVs.

\begin{tabular}{|c|c|c|c|c|c|c|c|c|c|c|c|c|c|c|}
\hline \multirow{2}{*}{$\begin{array}{c}\text { SEV } 1 \\
\text { Esp. } \\
\text { Cam.(m) }\end{array}$} & \multicolumn{2}{|c|}{$\begin{array}{c}\text { Coordenadas } \\
663746 / 8696012\end{array}$} & \multirow{2}{*}{\begin{tabular}{|c|} 
SEV 2 \\
$\begin{array}{c}\text { Esp. } \\
\text { Cam.(m) }\end{array}$
\end{tabular}} & \multicolumn{2}{|c|}{$\begin{array}{c}\text { Coordenadas } \\
665478 / 8694280\end{array}$} & \multirow{2}{*}{\begin{tabular}{|c|} 
SEV 3 \\
$\begin{array}{c}\text { Esp. } \\
\text { Cam.(m) }\end{array}$
\end{tabular}} & \multicolumn{2}{|c|}{$\begin{array}{c}\text { Coordenadas } \\
666718 / 8699173\end{array}$} & \multirow{2}{*}{\begin{tabular}{|c|} 
SEV 4 \\
$\begin{array}{c}\text { Esp. } \\
\text { Cam.(m) }\end{array}$
\end{tabular}} & \multicolumn{2}{|c|}{$\begin{array}{c}\text { Coordenadas } \\
669800 / 8694839 \\
\end{array}$} & \multirow{2}{*}{\begin{tabular}{|c|} 
SEV 5 \\
Esp. \\
Cam.(m)
\end{tabular}} & \multicolumn{2}{|c|}{$\begin{array}{c}\text { Coordenadas } \\
666308 / 8688087\end{array}$} \\
\hline & $\begin{array}{l}\text { resist. } \\
(\Omega . \mathrm{m})\end{array}$ & Geologia & & $\begin{array}{l}\text { resist. } \\
(\Omega . \mathrm{m})\end{array}$ & Geologia & & 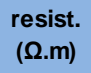 & Geologia & & $\begin{array}{l}\text { resist. } \\
(\Omega . \mathrm{m})\end{array}$ & Geologia & & $\begin{array}{l}\text { resist. } \\
(\Omega . \mathrm{m})\end{array}$ & Geologia \\
\hline 0.5 & 167.4 & \multirow{3}{*}{ Solo } & 0.5 & 153 & \multirow{3}{*}{ Solo } & 0.5 & 75.3 & \multirow{3}{*}{ Solo } & 0.5 & 184 & \multirow{3}{*}{ Solo } & 0.5 & 586 & \multirow{3}{*}{ Solo } \\
\hline 0.7 & 957.3 & & 1.1 & 947 & & 1 & 417.7 & & 0.15 & 1230 & & 0.4 & 4761 & \\
\hline 1.5 & 75.38 & & 3.4 & 78.6 & & 4.9 & 163.4 & & 0.95 & 71.3 & & 2.85 & 285 & \\
\hline 3.7 & 3394 & \multirow[t]{2}{*}{$\begin{array}{l}\text { Form. } \\
\text { Utiariti }\end{array}$} & 45 & 8260 & $\begin{array}{l}\text { Form. } \\
\text { Utiariti }\end{array}$ & 14.6 & 5988 & \multirow{2}{*}{$\begin{array}{l}\text { Form. } \\
\text { Utiariti }\end{array}$} & 7.4 & 1599 & \multirow{2}{*}{$\begin{array}{l}\text { Form. } \\
\text { Utiariti }\end{array}$} & 12.2 & 1022 & \multirow{2}{*}{$\begin{array}{l}\text { Form. } \\
\text { Utiariti }\end{array}$} \\
\hline 43.5 & 7112 & & 50 & 47.3 & \multirow{3}{*}{$\begin{array}{l}\text { Form. Salto } \\
\text { das Nuvens }\end{array}$} & 44 & 4814 & & 41 & 1688 & & 74 & 3999 & \\
\hline 350 & 134.9 & $\begin{array}{c}\text { Form. Salto } \\
\text { das } \\
\text { Nuvens }\end{array}$ & 75 & 325 & & 60 & 258.4 & \multirow{2}{*}{$\begin{array}{l}\text { Form. Salto } \\
\text { das Nuvens }\end{array}$} & 100 & 1387 & \multirow{2}{*}{$\begin{array}{c}\text { Form. Salto } \\
\text { das } \\
\text { Nuvens }\end{array}$} & 160 & 427 & \multirow{2}{*}{$\begin{array}{l}\text { Form. Salto } \\
\text { das Nuvens }\end{array}$} \\
\hline \multirow[t]{3}{*}{$>100$} & 1000 & \begin{tabular}{|c|} 
Form. \\
Dardanelos
\end{tabular} & 225 & 288 & & 200 & 304.4 & & 250 & 115.2 & & 150 & 466 & \\
\hline & & & $>100$ & 279 & $\begin{array}{c}\text { Form. } \\
\text { Dardanelos }\end{array}$ & 75 & 2978 & \multirow[t]{2}{*}{$\begin{array}{c}\text { Form. } \\
\text { Dardanelos }\end{array}$} & $>100$ & 1883 & $\begin{array}{c}\text { Form. } \\
\text { Dardanelos }\end{array}$ & $>100$ & 11276 & $\begin{array}{c}\text { Form. } \\
\text { Dardanelos }\end{array}$ \\
\hline & & & & & & $>100$ & 4558 & & & & & & & \\
\hline
\end{tabular}

\section{Conclusões}

A Técnica de Sondagem Elétrica Vertical permitiu distinguir, identificar e relacionar, através de uma análise quantitativa as camadas de diferentes resistividades elétricas aos horizontes de solos e substrato rochoso. Destacou-se precisamente a espessura da cobertura pedológica, a espessura e profundidade das formações Utiariti, Salto das Nuvens e Dardanelos.

\section{Referências}

Bhattacharya, P.K.; Patra, H.P, 1968. Direct Current Geoeletric Sounding. Elsevier

Company. - New York. 135p.

Publishing

Cutrim, A.O., Shiraiwa, S. 2011. Prospecção de água subterrânea no sudoeste do município de Rondonópolis (MT) usando sondagem elétrica vertical. Revista Brasileira de Geofísica, Rio de Janeiro, v. 29, n. 4, p. 45 51.

Cutrim, A.O., Rebouças A.C. 2005. Aplicação de sondagem elétrica vertical na estimativa do topo e da espessura de unidades geológicas da Bacia do Paraná na cidade de Rondonópolis-MT. Revista Brasileira de Geofísica. Rio de Janeiro, 23(1): 89-98.
Os dados gerados por esta pesquisa serão de grande importância para estimar as reservas de água dos Aquíferos Utiariti e Salto das Nuvens, avaliar a vulnerabilidade desses aquíferos, auxiliar na elaboração de projetos de poços para explorar esses aquíferos, assim como complementar o conhecimento da geologia da área e da região.

Cutrim, A.O., Ruiz. A.S.; Liporoni, L.M.; Medeiros. F.A.; Barroso, U.C. Nascimento, A.L. 2007. Sondagem Elétrica Vertical Aplicada em Pesquisa Hidrogeológica na bacia do Parecis, MT- Revista Brasileira de Geofísica, Vol. 25(2).

Padilha, A.V., Montes A. de S.L., Barros C.A.F. de, Lemos D.B., Frota G.B., Luz J. da S., Moreira J.M.P., Moreno J.J.P., Montes M.L., Menezes Filho N.R., Cardoso O.R.F.A., Lima P.F.C., Almeida W.J. de, Abreu Filho W., Silveira W.P., Santana W.R.R. 1974. Projeto Centro-Oeste de Mato Grosso. DNPM/CPRM, Goiânia, Relatório final, v. 1.

Prodeagro. 2001. Programa de Desenvolvimento do Agronegócio. Aspectos geológicos da Folha Rio Claro MIR 356 (SD.21-X-C). 235 p. 
Ribeiro, D.B.S. 2009. Avaliação hidrogeológica na cidade de Sinop - MT. Trabalho de Conclusão de Curso (Bacharelado em Geologia). Instituto de Ciências Exatas e da Terra. Departamento de Geologia Geral. Universidade Federal de Mato Grosso, Cuiabá.
Tikhonov, A.N.; Arsenin, V.Y. 1977. Solutions of ill-posed problems. Winston \& Sons, New York, 349 p. 